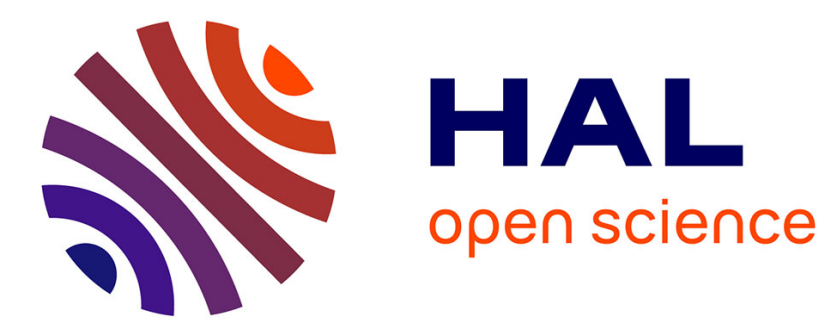

\title{
Bistable [ c 2] Daisy Chain Rotaxanes as Reversible Muscle-like Actuators in Mechanically Active Gels
} Antoine Goujon, Thomas Lang, Giacomo Mariani, Emilie Moulin, Gad Fuks, Jesus Raya, Eric Buhler, Nicolas Giuseppone

\section{- To cite this version:}

Antoine Goujon, Thomas Lang, Giacomo Mariani, Emilie Moulin, Gad Fuks, et al.. Bistable [ c 2] Daisy Chain Rotaxanes as Reversible Muscle-like Actuators in Mechanically Active Gels. Journal of the American Chemical Society, 2017, 139 (42), pp.14825-14828. 10.1021/jacs.7b06710 . hal03507961

\section{HAL Id: hal-03507961 \\ https://hal.science/hal-03507961}

Submitted on 3 Jan 2022

HAL is a multi-disciplinary open access archive for the deposit and dissemination of scientific research documents, whether they are published or not. The documents may come from teaching and research institutions in France or abroad, or from public or private research centers.
L'archive ouverte pluridisciplinaire HAL, est destinée au dépôt et à la diffusion de documents scientifiques de niveau recherche, publiés ou non, émanant des établissements d'enseignement et de recherche français ou étrangers, des laboratoires publics ou privés. 


\title{
Bistable [c2]daisy chain rotaxanes as reversible muscle-like actuators in mechanically active gels
}

\author{
Antoine Goujon, ${ }^{[\mathrm{a}]}{ }_{\uparrow}$ Thomas Lang, ${ }^{[\mathrm{a}]}$ Giacomo Mariani, ${ }^{[\mathrm{b}]}$ Emilie Moulin, ${ }^{[\mathrm{a}]}$ Gad Fuks, ${ }^{[\mathrm{a}]}$ Jesus Raya, ${ }^{[\mathrm{c}]}$ \\ Eric Buhler, ${ }^{[\mathrm{b}]}$ and Nicolas Giuseppone ${ }^{[\mathrm{a}]^{*}}$ \\ ${ }^{\text {[a] }}$ SAMS research group - University of Strasbourg - Institut Charles Sadron, CNRS - 23 rue du Loess, BP 84047, 67034 \\ Strasbourg Cedex 2, France. \\ ${ }^{[b]}$ Matière et Systèmes Complexes (MSC) Laboratory, UMR CNRS 7057, Sorbonne Paris Cité, University of Paris Diderot- \\ Paris VII, Bâtiment Condorcet, 75205 Paris Cedex 13, France. \\ ${ }^{[c]}$ Institut de Chimie, UMR 7177 CNRS, Université de Strasbourg, 1 rue Blaise Pascal, BP 296 R8, 67008 Strasbourg Cedex.
}

\section{Supporting Information Placeholder}

\begin{abstract}
The implementation of molecular machines in polymer science is of high interest to transfer mechanical motions from nanoscale to macroscale in order to access new kinds of active devices and materials. Towards this objective, thermodynamic and topological aspects need to be explored for reaching efficient systems capable of producing a useful work. In this paper we describe the branched polymerization of $\mathrm{pH}$-sensitive bistable [c2]daisy chain rotaxanes by using copper (I) catalyzed Huisgen 1,3-dipolar cycloaddition ("click chemistry"). With this cross-linked topology, the corresponding materials in the form of chemical gels can be contracted and expanded over a large variation of volume $(\approx 50 \%)$ by changing the protonation state of the system. HR-MAS ${ }^{1} \mathrm{H}$ NMR and neutron scattering experiments reveal that this macroscopic response of the gels result from the synchronized actuation of the mechanical bonds at the molecular level.
\end{abstract}

In living systems, the integration in space and time of protein machineries with the dynamic structuring of the cytoskeleton is central to many functions such as growth, motility, contraction, and mechanotransduction. ${ }^{1,2}$ These important biological responses can be modelled as a mechanical continuum going from subcellular components to tissues and organs. ${ }^{3,4}$ Partly inspired by biomolecular machines, ${ }^{5,6}$ chemists have synthesized a number of artificial molecules capable of relative internal translations or rotations. ${ }^{7-9}$ Their individual functioning can be actuated at thermodynamic equilibrium (molecular switches) ${ }^{10,11}$ or out-of-equilibrium (molecular motors). ${ }^{12-16}$ However, to make use of the work produced by these nanomachines, one should link them to other elements which can amplify and/or transduce their mechanical motion in something useful. ${ }^{17-20}$ Following these lines, a few recent works have demonstrated the possible integration of large numbers of artificial molecular machines in order to connect them with the outside world. ${ }^{21-25}$

A particularly interesting class of artificial nanomachines to be further coupled in space and time are bistable [c2]daisy chain rotaxanes (so-called "molecular muscles"). ${ }^{26}$ Their complex molecular structure involves two rings linked to two axles that are double threaded (Figure 1a). By introducing different binding stations for the macrocycles on the axles, the controlled gliding of their subunits can lead to internal contraction and extension based on the dynamic of their mechanical bond. ${ }^{27-30}$ The first covalent oligomers based on [c2] daisy chain rotaxanes have been described independently by the groups of Stoddart ${ }^{31}$ and Grubbs. ${ }^{32}$ Our group recently introduced coordination ${ }^{33}$ and hydrogen-bonded ${ }^{34-36}$ main chain supramolecular polymers involving $\mathrm{pH}$-responsive bistable [c2]daisy chain rotaxanes (Figure 1b). In these examples, high degrees of polymerization were demonstrated, leading for instance to i) microscopic extension / contraction of single chain polymers; ${ }^{33}$ ii) supramolecular polymerization / depolymerization process; ${ }^{36}$ iii) changes of mesophase morphologies in bundles of supramolecular polymers $;{ }^{35}$ and $i v$ ) macroscopic sol-gel transitions in supramolecular polymer networks. ${ }^{34}$ Another important advance was made by the group of Harada who synthesized a covalent 3D polymer network integrating [ $c 2$ ]daisy chains in which cyclodextrines (rings) bind to photoswitchable azobenzene groups (axles). ${ }^{37}$ Of peculiar interest, in the dry state, the corresponding xerogel is able to perform a fast pseudo-reversible deformation induced by an alternation of UV and visible lights.

a)

[c2] daisy chain monomers

c) Cross-linked polymer

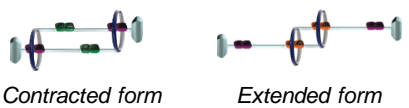

b)
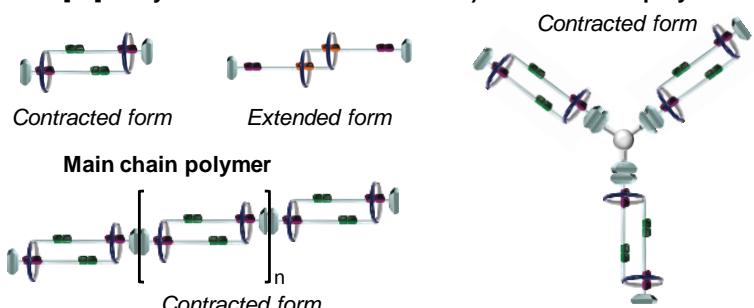

d)

$$
\text { Contracted form }
$$

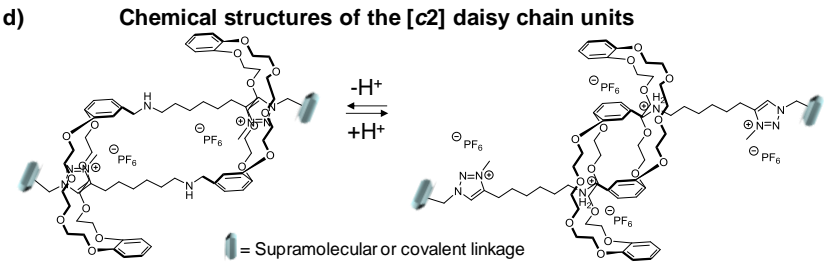

Figure 1. a) Schematic representation of contracted and extented [c2] daisy chain monomers; b) Schematic representation of a main chain polymer based on [c2] daisy chain monomers; c) Schematic representation of a cross-linked polymer network based on [c2] daisy chain monomers; d) Detailed chemical structure of the [c2] daisy chain unit used in the present study. 
In the present article, we now explore a branched covalent polymer involving $\mathrm{pH}$-sensitive bistable [ $c 2$ ] daisy chain rotaxanes as monomers (Figures 1c,d). We show that in the chemically crosslinked network topology, the $\mathrm{pH}$-actuation of the mechanical bond at the molecular level translates to large and fully reversible macroscopic contraction/expansion of the swollen chemical gel by $\approx$ $50 \%$ of its volume.

The synthetic design of the present chemical network is depicted in Figure 2a. It involves the cross-linking by click chemistry of three components: pseudo rotaxane bis-alkyne $\mathbf{1},{ }^{33,38}$ bis-azide $\mathbf{2}$, and tris-azide $\mathbf{3}$ (see SI for the detailed syntheses and characterization of compounds $\mathbf{2}$ and $\mathbf{3}$ ). We used three different proportions of cross-linking agent 3 (Figure $2 b$ ) and the reactions were performed directly into a metallic mold (Figure S1). This protocol led to the formation within 30 minutes of plastic-like materials $\left(\mathbf{G e l 4 ^ { A - C }}\right)$ which swell upon addition of acetonitrile. $\mathbf{G e l 4 ^ { \mathbf { A } }}$ and $\mathbf{G e l 4}^{\mathbf{B}}$ were robust enough to be easily manipulated, while $\mathbf{G e l 4 ^ { C }}$ was too soft and fragile to be further studied. Traces of remaining starting materials were removed by several rinsing of $\mathbf{G e l} 4^{\mathbf{A}}$ and $\mathbf{G e l} \mathbf{4}^{\mathrm{B}}$ in dichloromethane, and copper salts were then extracted using an aqueous solution of ethylenediaminetetraacetic acid (EDTA) at a $\mathrm{pH}$ of 8. The triazole functions in $\mathbf{G e l 4 ^ { \mathbf { A } }}$ and $\mathbf{G e l 4}^{\mathbf{B}}$ were finally methylated to furnish $\mathbf{G e l 4}^{\mathrm{AM}}$ and $\mathbf{G e l 4}{ }^{\mathrm{BM}}$ by simple immersion of the gels in a solution of acetonitrile and methyl iodide (1:1) followed by anion exchange with ammonium hexafluorophosphate. After rinsing and evaporation of the solvents, the plastic materials can be reswollen in acetonitrile up to $1900 \%$ of their dry volume (Figure S2).

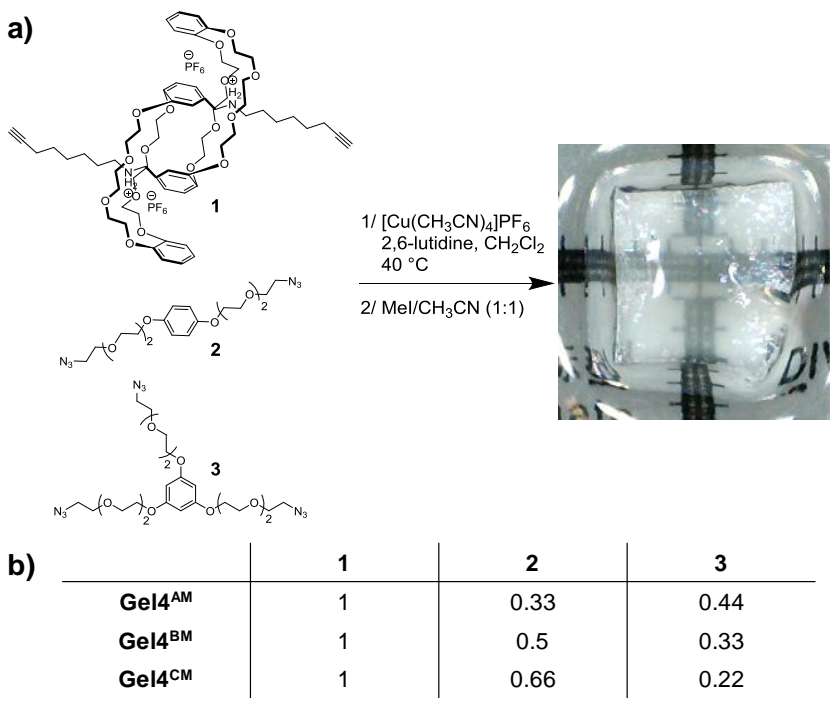

Figure 2. a) Chemical structures of monomers 1-3 and experimental conditions leading to the formation of chemical gels as illustrated by the picture on the right; b) Compositions and labels of the gels obtained using the conditions described in panel (a) and for different ratios of monomers.

We then probed the contraction/expansion of the material by direct macroscopic observation. The process was performed stepwise in order to swell the gel in the same conditions when contracted or expanded, and thus to avoid solvation effects. In their initial state, the gels were swollen in acetonitrile and the [c2]daisy chains were in their protonated (extended) state. The initial pieces of Gel4 ${ }^{\mathrm{AM}}$ and $\mathbf{G e l 4}{ }^{\mathbf{B M}}$ were measured before being immersed in a $1 \mathrm{M}$ solution of sodium hydroxide to deprotonate the [c2]daisy chains units. The resulting gels were rinsed and swollen back in acetonitrile to measure their volumes. The contracted pieces of gels were then immersed in an aqueous solution of ammonium hexafluorophosphate to protonate the $[c 2]$ daisy chains, and they were again rinsed and swollen back in acetonitrile before measuring their volumes (Figure $3 \mathrm{a}-\mathrm{c})$. We used the software ImageJ to evaluate the size change of the main surface area of the gel after each step. As the contraction and expansion are isotropic in this random network configuration, the following equation was used to estimate the volume change: $V_{\text {rel }}=\left(A_{f} / A_{i}\right)^{3 / 2}$, where $V_{\text {rel }}$ being the relative volume in $\%$, $A_{f}$ the surface area after protonation or deprotonation, and $A_{i}$ the surface area before starting the experiment. As shown in Figure 3d, the volume of Gel4 ${ }^{\mathrm{AM}}$ changed from $100 \%$ to $68 \%$ after deprotonation, and went back to $110 \%$ after protonation. The volume of Gel4 ${ }^{\mathbf{B M}}$ decreased from $100 \%$ to $61 \%$ after deprotonation and went back to $98 \%$ after protonation. Repetitive sequences of protonation and deprotonation were performed on gel Gel4 ${ }^{\mathrm{AM}}$, showing the successful cycling of contraction and expansion of the material at least three times without degradation (Figure 3f). The fact that the sizes slightly exceed the original sizes after the first cycle can be explained by the partial exchange of counter anions of the [c2]daisy chains during the experiments, modifying their swelling ability. In both cases, the volume variations measured are in good agreement with the expected response of the [c2]daisy chain units, which strongly suggest their successful incorporation in the materials and their dynamic integration from nano- to macroscopic scales. Moreover, control experiments with non-methylated gels Gel4 ${ }^{\mathbf{A}}$ and $\mathbf{G e l}^{\mathbf{B}}$ showed only negligible variations of their volumes after using the same protocol of deprotonation/protonation: 100-95-101\% for $\mathbf{G e l 4}^{\mathbf{A}}$ and $\mathbf{1 0 0 - 9 9 - 1 0 0 \%}$ for $\mathbf{G e l 4}^{\mathbf{B}}$ (Figure 3e). Importantly, this result confirms that the presence of a second station for the rotaxane (i.e. the triazolium binding site) is mandatory to observe contraction and expansion of the material. It also indicates that the protonation / deprotonation of the secondary amine does not lead to a measurable change in the swelling properties of the gel. ${ }^{39} \mathrm{Fi}-$ nally, we performed a HR-MAS ${ }^{1} \mathrm{H}$ NMR experiment ${ }^{40}$ which allowed us to directly probe the motion of the mechanical bond in the gel state for Gel4 ${ }^{\mathbf{A M}}$ (Figure S7). The typical $0.5 \mathrm{ppm}$ downfield shift of the triazolium proton resonance signal in the contracted form ( $v s$. upfield shift in the extended form) unambiguously proved the expected actuation in the gel state. Altogether, these experiments illustrate the relationship existing between the volume variations at the macroscopic scale and the controlled dynamic of the [c2]daisy chains at the molecular level.

To probe further the conformational dynamic at the levels of the mechanical bond and of the polymer chain, we performed small angle neutron scattering (SANS) experiments. Figure 4a shows variations in absolute scattered intensity, $I$, as a function of the magnitude of the scattering wave vector, $q$, for both expanded and contracted Gel4 ${ }^{\mathrm{AM}}$. In the experimental scattering vector regime $q>$ $R_{G}^{-1}$, where $R_{\mathrm{G}}$ is the radius of gyration of the objects, the scattered intensity of a fractal particle is given by $I(q) \approx q^{-D_{f}}$. Here, $D_{f}$ is the so-called fractal dimension of the particle. Both scattering curves in Figure 4a exhibit an overall behavior characterized by the following sequence: $(i)$ a power law regime at low $q$ giving a fractal exponent $D_{f}=3$ and indicating the presence of large assemblies; (ii) another power law regime in the mid $q$ region with $D_{f}=1$ and characteristic of the rod-like behavior of the chains for distances smaller than the persistence length; and finally (iii) a Guinier regime at high $q$ associated to the cross-section of the chains. The signal measured at mid and high $q$ corresponds to the scattering of the polymer chains that compose the network. The two curves decay at the same $q$ in the high $q$ region. This means that the crosssection remains almost unchanged in the extended and contracted state and that there is no bundling of the polymer chains when switching the system. 

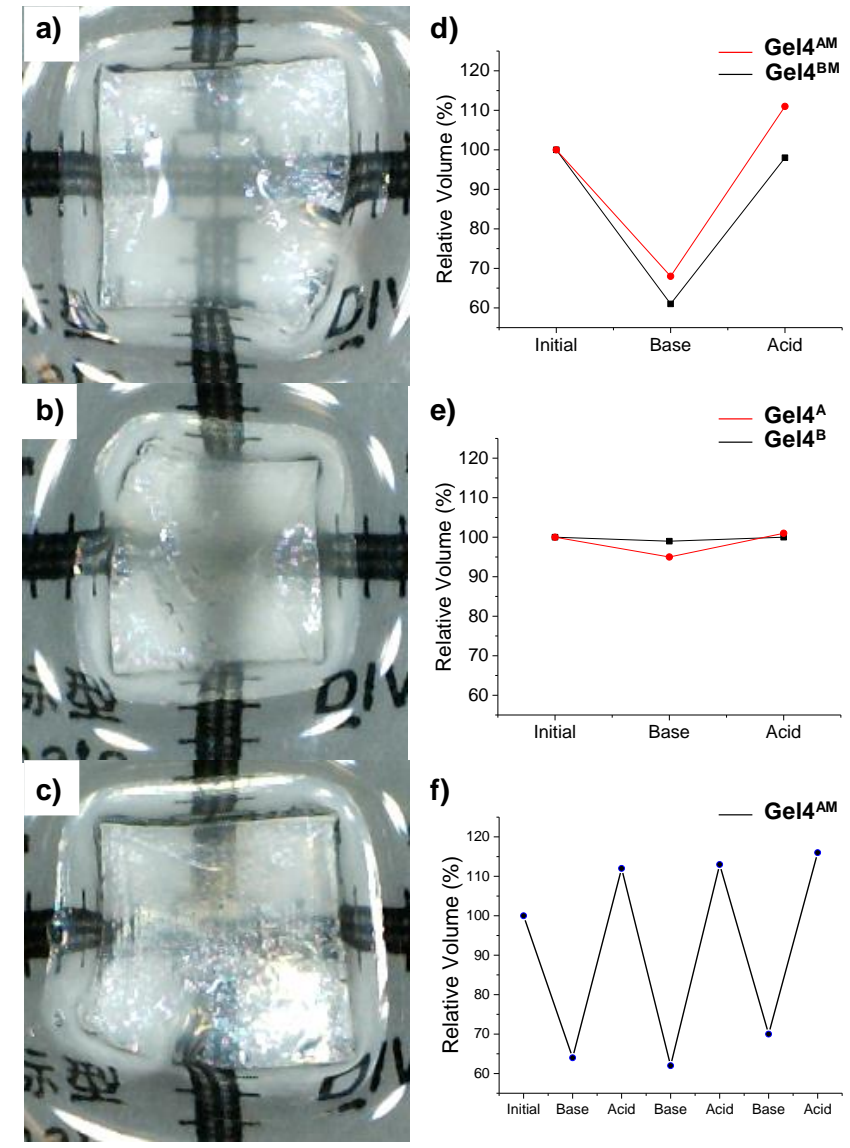

Figure 3. a-c) Pictures of methylated gels $4^{\text {GelAM }}$ (a), after treatment with $\mathrm{NaOH}$ (b) and after being subsequently treated with $\mathrm{NH}_{4} \mathrm{PF}_{6}(\mathbf{c})$; d) Variation of volume $V_{\text {rel }}$ upon contraction/expansion for methylated gels $\mathbf{4}^{\text {GelAM }}$ and $\mathbf{4}^{\text {GelBM}}$; e) Variation of volume $V_{\text {rel }}$ upon contraction/expansion for non-methylated gels $\mathbf{4}^{\text {GelA }}$ and $4^{\mathrm{GelB}}$; f) Variation of volume $V_{\text {rel }}$ upon three contraction/expansion cycles for methylated gels $\mathbf{4}^{\text {GelAM }}$.

The Guinier approximation for elongated objects yields a crosssection radius of gyration of $R_{\mathrm{c}}=0.47$ and $0.50 \pm 0.03 \mathrm{~nm}$ for extended and contracted $\mathbf{G e l} \mathbf{4}^{\mathbf{A M}}$, respectively. In the mid $q$ region, both curves increase proportionally to $q^{-1}$ but, their intensity is markedly different (Figure $4 \mathrm{~b}$ ). This observation is extremely important since the signal intensity is only proportional to the linear mass density $M_{\mathrm{L}}$ of the polymer chain in this region (see more details in SI section 5), and a higher intensity corresponds to a higher linear mass density. Hence, the contracted system has a higher linear mass density as compared to the extended one and this clearly proves the contraction of the [ $c 2]$ daisy chains composing the polymer network. This behavior is in full agreement with previously reported literature on single chain polymers. ${ }^{33}$ The data in this regime of $q$ range can be fitted satisfactorily using a rigid-rod model ${ }^{41}$ giving a $M_{\mathrm{L}}=325 \mathrm{~g} \mathrm{~mol}^{-1} \mathrm{~nm}^{-1}$ for the extended polymer, and a new $M_{\mathrm{L}}=480 \mathrm{~g} \mathrm{~mol}^{-1} \mathrm{~nm}^{-1}$ for the contracted one (+50\%) (Figure 4b). This compares well with the theoretical linear mass densities $M_{\mathrm{L}}$ of 365 and $456 \mathrm{~g} \mathrm{~mol}^{-1} \mathrm{~nm}^{-1}$ for the extended and the contracted, respectively (see SI section 5). For the present system we were not able to discriminate the persistence length of the two polymers because the transition between the $q^{-1}$ rod-like and the $q^{-2}$ coil-like characteristic regimes, observed for polymer chains, ${ }^{41}$ is here masked by the $q^{-3}$ upturn at low $q$. However, the transition between the $q^{-1}$ and the $q^{-3}$ regimes occurring at $q^{*}$ can give a lower bound for the persistence length that is $l_{\mathrm{P}} \geq 1.91 / q^{*} \sim 13 \pm 2 \mathrm{~nm} .{ }^{41}$ The reason for which, at low $q$, the signal rises proportionally to $q^{-3}$ is the presence of heterogeneities in the network with size larger than the investigated $q$ range; i.e., larger than $40 \mathrm{~nm}$.

The work reported here shows the integration of $\mathrm{pH}$ switchable [c2]daisy chain rotaxanes in "clicked" polymer networks. In this topology, the resulting chemical gels can be swollen by 20 times of their volume and produce cyclic muscle-like contraction and expansion upon protonation and deprotonation. Such a macroscopic response was not accessible to date by using this type of bistable [c2]daisy chain, neither by incorporating them in linear polymers ${ }^{31,33,35,42}$ or in physical gels which could not sustain their topological integrity upon mechanical work. ${ }^{34}$ Importantly, HR-MAS ${ }^{1} \mathrm{H}$ NMR in the gel state and control experiments performed by removing the triazolium station in the [c2]daisy chain show that the macroscopic change in volume is directly related to the actuation of the mechanical bond at the molecular level. SANS experiments confirmed this relationship by showing a 50\% increase of the linear mass density of the polymer chains in the network upon contraction, while their cross-section remains constant. The understanding of such a large amplification of the conformational dynamic of polymer chains in network topologies up to the macroscopic response of a gel is of high interest for the implementation of molecular machines in materials science.
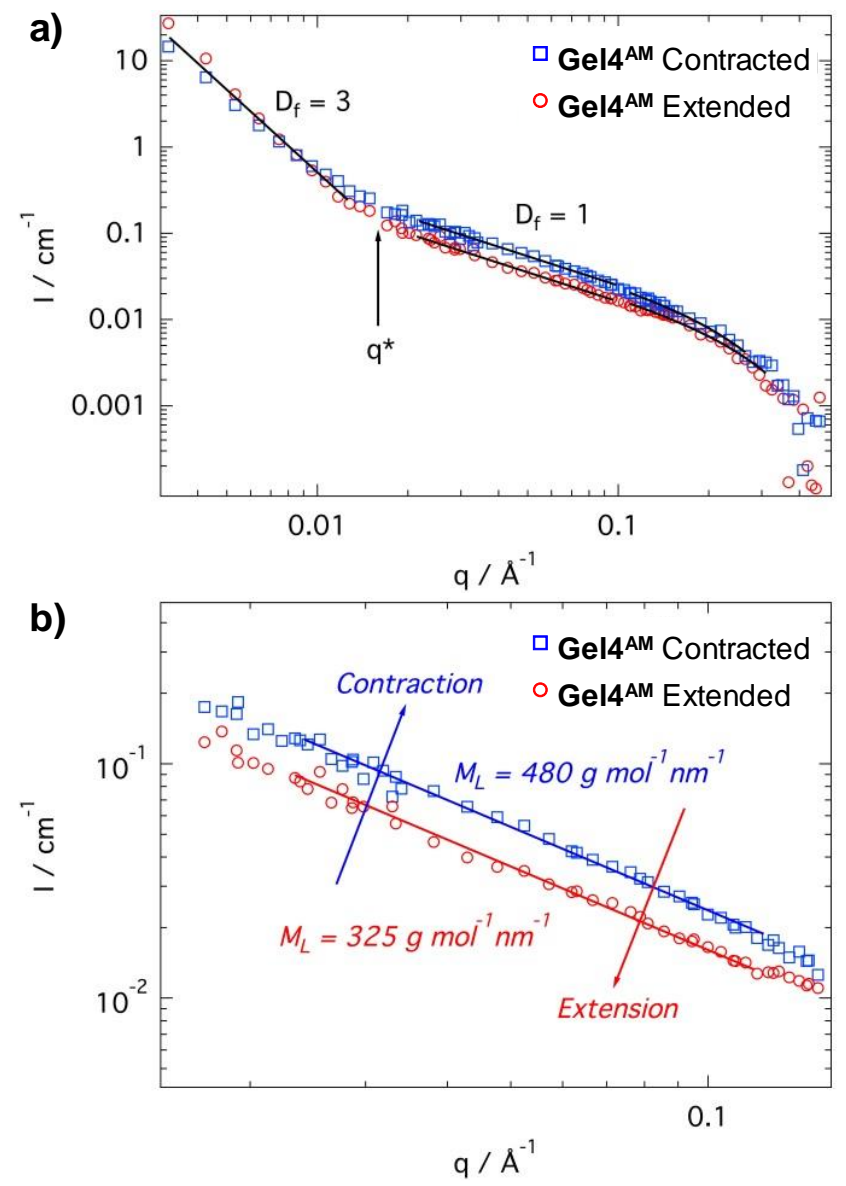

Figure 4. a) SANS curves for Gel4 ${ }^{\mathrm{AM}}$ contracted and Gel4 ${ }^{\mathrm{AM}}$ extended polymers. The high $q$ data have been fitted by a Guinier expression for the form factor of the polymer cross-section (see continuous line and eq 4 in SI). The arrow indicates the transition $q^{*}$ between the $q^{-1}$ and $q^{-3}$ domains. b) Enlargement of the $q$-range demonstrating the difference in linear mass density between the two polymers. The continuous lines represent the best fits of the data using a rigid rod model (see SI, eq 5). 


\section{ASSOCIATED CONTENT}

\section{Supporting Information}

Synthetic protocols and characterization of compounds $\mathbf{2}$ and $\mathbf{3}$, synthetic protocols for the formation of gels, procedures for the contraction and extension experiments, SANS experimental procedures. This material is available free of charge via the Internet at http://pubs.acs.org.

\section{AUTHOR INFORMATION}

\section{Corresponding Author}

giuseppone@unistra.fr

\section{Present Addresses}

$\uparrow$ Current address: Department of Organic Chemistry, University of Geneva, 30 Quai Ernest Ansermet, 1211 Geneva 4, Switzerland Notes

The authors declare no competing financial interests.

\section{ACKNOWLEDGMENTS}

This work was supported by the Agence Nationale pour la Recherche (Grant number ANR-14-CE06-0021, fellowships to A.G., T.L. and G.M.) and the French Ministry of Research (fellowship to A.G.). We wish to thank the Laboratory of Excellence for Complex System Chemistry (LabEx CSC), the international center for Frontier Research in Chemistry (icFRC), the Centre National de la Recherche Scientifique (CNRS), the University of Strasbourg (Unistra), the University of Paris Diderot (Sorbonne Paris Cité) and the Institut Universitaire de France (N.G.) for financial supports. We also thank the Laboratoire Léon Brilloin (LLB, CEA, Saclay, France) for beamtime allocation and Dr. Jean-Marc Strub for HRMS experiments.

\section{REFERENCES}

(1) Schliwa, M.; Woehlke, G. Nature 2003, 422, 759.

(2) Vale, R. D. Science 2000, 288, 88.

(3) Bao, G.; Suresh, S. Nat. Mater. 2003, 2, 715.

(4) Wang, N.; Naruse, K.; Stamenovic, D.; Fredberg, J. J.; Mijailovich, S. M.; Tolic-Norrelykke, I. M.; Polte, T.; Mannix, R.; Ingber, D. E. Proc. Natl. Acad. Sci. 2001, 98, 7765.

(5) van den Heuvel, M. G. L.; Dekker, C. Science 2007, 317, 333.

(6) Kinbara, K.; Aida, T. Chem. Rev. 2005, 105, 1377.

(7) Coskun, A.; Banaszak, M.; Astumian, R. D.; Stoddart, J. F.; Grzybowski, B. A. Chem. Soc. Rev. 2012, 41, 19

(8) Abendroth, J. M.; Bushuyev, O. S.; Weiss, P. S.; Barrett, C. J. ACS Nano 2015, 9, 7746.

(9) Erbas-Cakmak, S.; Leigh, D. A.; McTernan, C. T.; Nussbaumer, A. L. Chem. Rev. 2015, 115, 10081.

(10) Xue, M.; Yang, Y.; Chi, X.; Yan, X.; Huang, F. Chem. Rev. 2015, 115,7398 .

(11) Tian, H.; Wang, Q.-C. Chem. Soc. Rev. 2006, 35, 361.

(12) Wilson, M. R.; Solà, J.; Carlone, A.; Goldup, S. M.; Lebrasseur, N.; Leigh, D. A. Nature 2016, 534, 235.

(13) Ragazzon, G.; Baroncini, M.; Silvi, S.; Venturi, M.; Credi, A. Nat. Nanotechnol. 2015, 10, 70 .

(14) Cheng, C.; McGonigal, P. R.; Schneebeli, S. T.; Li, H.;

Vermeulen, N. A.; Ke, C.; Stoddart, J. F. Nat. Nanotechnol. 2015 $10,547$.

Greb, L.; Lehn, J.-M. J. Am. Chem. Soc. 2014, 136, 13114.

Balzani, V.; Clemente-Leon, M.; Credi, A.; Ferrer, B.; Venturi, M.; Flood, A. H.; Stoddart, J. F. Proc. Natl. Acad. Sci. 2006, 103, 1178

Bléger, D. Macromol. Chem. Phys. 2016, 217, 189.

Wang, J.; Feringa, B. L. Science 2011, 331, 1429.

Green, J. E.; Wook Choi, J.; Boukai, A.; Bunimovich, Y.; Johnston-Halperin, E.; DeIonno, E.; Luo, Y.; Sheriff, B. A.; Xu, K.; Shik Shin, Y.; Tseng, H.-R.; Stoddart, J. F.; Heath, J. R. Nature 2007, 445, 414

Lin, Q.; Hou, X.; Ke, C. Angew. Chem. Int. Ed. 2017, 56, 4452. Berna, J.; Leigh, D. A.; Lubomska, M.; Mendoza, S. M.; Perez, E. M.; Rudolf, P.; Teobaldi, G.; Zerbetto, F. Nat Mater 2005, 4 , 704.

Huang, T. J.; Brough, B.; Ho, C.-M.; Liu, Y.; Flood, A. H.; Bonvallet, P. A.; Tseng, H.-R.; Stoddart, J. F.; Baller, M.; Magonov, S. Appl. Phys. Lett. 2004, 85, 5391.

Eelkema, R.; Pollard, M. M.; Vicario, J.; Katsonis, N.; Ramon, B. S.; Bastiaansen, C. W. M.; Broer, D. J.; Feringa, B. L. Nature 2006, 440, 163.

Li, Q.; Fuks, G.; Moulin, E.; Maaloum, M.; Rawiso, M.; Kulic, I.; Foy, J. T.; Giuseppone, N. Nat. Nanotechnol. 2015, 10, 161.

Foy, J. T.; Li, Q.; Goujon, A.; Colard-Itté, J.-R.; Fuks, G.; Moulin, E.; Schiffmann, O.; Dattler, D.; Funeriu, D. P.; Giuseppone, N. Nat. Nanotechnol. 2017, 12, 540

Jiménez, M. C.; Dietrich-Buchecker, C.; Sauvage, J.-P. Angew. Chem. Int. Ed. 2000, 39, 3284.

Bruns, C. J.; Stoddart, J. F. Acc. Chem. Res. 2014, 47, 2186.

Kay, E. R.; Leigh, D. A. Angew. Chem. Int. Ed. 2015, 54, 10080. Stoddart, J. F. Chem. Soc. Rev. 2009, 38, 1802.

Bruns, C. J.; Stoddart, J. F. The Nature of the Mechanical Bond: From Molecules to Machines; John Wiley \& Sons, Inc.: Hoboken, NJ, USA, 2016.

Fang, L.; Hmadeh, M.; Wu, J.; Olson, M. A.; Spruell, J. M.; Trabolsi, A.; Yang, Y. W.; Elhabiri, M.; Albrecht-Gary, A. M.; Stoddart, J. F. J. Am. Chem. Soc. 2009, 131, 7126.

Clark, P. G.; Day, M. W.; Grubbs, R. H. J. Am. Chem. Soc. 2009, 131,13631 .

Du, G.; Moulin, E; Jouault, N.; Buhler, E.; Giuseppone, N. Angew. Chem. Int. Ed. 2012, 51, 12504.

Goujon, A.; Mariani, G.; Lang, T.; Moulin, E.; Rawiso, M.; Buhler, E.; Giuseppone, N. J. Am. Chem. Soc. 2017, 139, 4923.

Goujon, A.; Du, G.; Moulin, E.; Fuks, G.; Maaloum, M.; Buhler, E.; Giuseppone, N. Angew. Chem. Int. Ed. 2016, 55, 703.

Wolf, A.; Moulin, E.; Cid Martín, J. J.; Goujon, A.; Du, G.; Busseron, E.; Fuks, G.; Giuseppone, N. Chem. Commun. 2015, 51,4212 .

Iwaso, K.; Takashima, Y.; Harada, A. Nat. Chem. 2016, 8, 625. Romuald, C.; Busseron, E.; Coutrot, F. Org. Lett. 2008, 10, 3741. Here the secondary ammonium site is shielded by the strong chelation to the macrocycle and does not present the swelling behavior of some polyelectrolytes in water. For $\mathrm{pH}$-sensitive cationic hydrogels see for instance: Ramos, J.; Forcada, J.; Hidalgo-Alvarez, R. Chem. Rev. 2014, 114, 367.

Andrew, E. R.; Bradbury, A.; Eades, R. G. Nature 1958, 182, 1659.

Buhler, E.; Boué, F. Macromolecules 2004, 37, 1600

Gao, L.; Zhang, Z.; Zheng, B.; Huang, F. Polym. Chem. 2014, 5 , 5734 . 


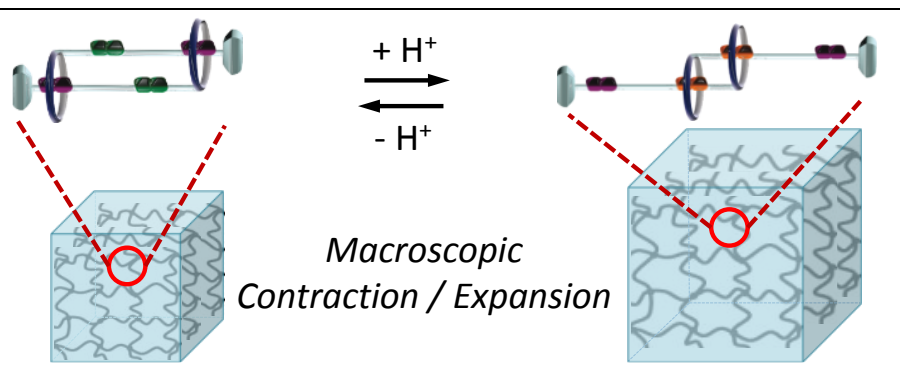

